\title{
Dynamic sub-mesh protection under dynamic traffic demands in dense WDM networks
}

\author{
Chen-Shie Ho \\ National Taiwan University \\ hocs@lion.ee.ntu.edu.tw
}

\author{
Ing-Yi Chen \\ National Taipei University of \\ Technology \\ ichen@csie.ntut.edu.tw
}

\author{
Sy-Yen Kuo \\ National Taiwan University \\ sykuo@cc.ee.ntu.edu.tw
}

\begin{abstract}
Network survivability is a key issue in reliable WDM optical network design to assure the service guarantee to customers. Rather than conventional connection link/ path/sub-path based protection switching scheme, in this paper we propose protection group (sub-mesh)-based approach to fast recover traffic from single/multiple link/node failures. By the group-based spare resource discovery and exploitation, the local channel utilization of network will be maximized and hence enhance the overall quality of protection. The simulation results show that the local resource utilization and restoration time are efficient under heavy load in the dynamic traffic environment over dense mesh backbone networks.
\end{abstract}

\section{Introduction}

WDM networks carry high volumes of traffic loads, and failures may result in severe loss, especially in business transaction. Therefore, it is imperative that these networks are implemented by effective fault tolerance mechanisms, that is, the ability of a network to reconfigure and re-establish original communication upon failure, to minimize the huge avenue loss [1]. This paper will mainly consider the dynamic traffic condition on the wide area wavelength routed mesh topology based backbone environment. The survivability mechanisms against link/node failure in optical layer can be divided to preplanned protection scheme and dynamic restoration scheme. The restoration methods can be further classified into reactive and proactive methods [3][4][5]. These methods are either link-based or path-based [2][3][4]. Besides the link and path protection, there are other protection switching methods exist in the literatures [9][10]. Recently the sub-path or segment protection strategy was proposed [6][7][8]. The main idea is dividing a given working path into overlapped segments while protecting each segment separately. In [7], a variation of sub-path protection is presented which implemented the network partition to protect the working lightpath segment in each area. It has advantages in multiple failure recovery, ILP scalability and fast recovery time, and suitable for low connection loading condition. When the number of traffic demands increases, the capacity of each area should be adjusted to fit new spare capacity requirements. In [8] a segment partition method under the short leap shared protection (SLSP) framework is proposed. The SLSP provides finer service granularity and higher network throughput, and several algorithms were proposed to perform segment allocation and path selection to realize SLSP. However, all the proposed ideas mainly work on a per-connection basis.

\section{Group protection switching}

We illustrate these concepts in Figure 1. Suppose there is a connection request between (source, destination) $=$ (A,D). After setup process the working path is determined to A-F-E-D. If the path-based proactive strategy is chosen to survive the failure event occurred in link E-F or any link located on the working path, the protection or spare path A-B-C-D will be replaced to continue the traffic after short reconfiguring time. By link-based proactive scheme, the spare path remains as working path except the failure portion E-F, the end-nodes on both sides of failure link will find an alternate (shortest) path to reconnect them and complete the connection. The rerouting complexity of linkbased scheme is lower than path-based approach but it also lacks of the flexibility the path-based scheme has. If we can relax the selection extent to a more flexible environment choosing by some resource-oriented criteria, there maybe a better efficiency and survivability can be achieved. As the same example in Fig.1, if one chooses the protection group A-B-C-D-E-F-A as the resource pool for any failure restoration located within this area, A-F-B-C-D will be the spare path if there are nore available network capacity existed in GD than C-E or ED. Our approach uses this concept (we called group protection) with link state protocol that is used to distribute topology information about the network to form a new protection scenario. The objective of the group finding algorithm is to determine the suitable protection domain compassing by the working path and protection path based on the 
weighting function. This protection groups will provide necessary backup resource for successive reliable connection requests. The group switching schemes can be further divided into 2 categories: static and dynamic grouping, which will be discussed later in section 3 and 4 , respectively.

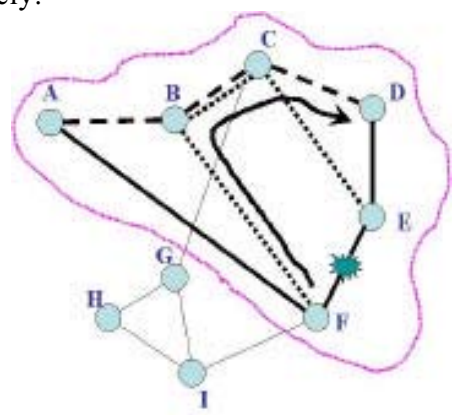

Figure 1. Illustration of link, path and groupbased protection.

\section{Static grouping protection}

In static grouping scenario, the whole network will be partitioned in several fixed-size groups. The boundary location and the dimension of the group will be determined depending on the network topology, the traffic patterns and the protection strategies. In general, from viewpoint of the network topology, k-connect graph partition and average node degree partition can be used as the partition metrics. For different protection demands, different protection means can be used.

We apply the criteria on designing the static grouping protection scheme to the exampled Italian network, which is shown in Fig. 2. There are 16 connection requests distributed on different node pairs with uniform distribution. The average hop number of connection is 2.1, which makes the produced group seems small. As seen from the figure, the minimum $\mathrm{k}$-connect group finding with nodal connectivity consideration ensure the single link/node failure occurred in each of the group. The overlapped area will be shared by adjacent group for flexible spare resource allocation. The group size and partition with sharability will change under different traffic patterns and quality of protection demands. The optimal group partition for resource usage can be found by ILP formations derived for static traffic situations.

\section{Dynamic grouping protection}

It is known that ILP formulations of RWA problems own high computational complexity. The large number of variables and constraints generated made it only possible properly applied to small scaled networks. Also, the static grouping method is suitable for static traffic environment because of its fixed resource allocation. In dynamic traffic condition, the connection requests arrive in a random manner. When a new reliable-demand connection request arrives, we find the working path and protection path simultaneously by two node-disjoint paths to protect against single link/node failure. We assume that all of the routes between one node to another are stored in the node.

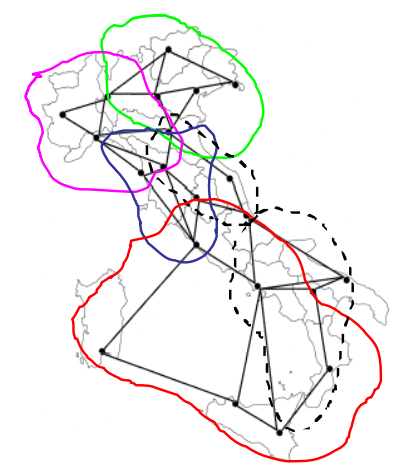

Figure 2. Result of partitioned protection group in sample network.

So the working-protection paths can be taken just from the routes database. It will first find a feasible path as the working path, then use the weighting function as follows to find the protection path:

weight $=\alpha \times$ hopcount $+\beta \times$ availablevavelengh in a link +

$$
\gamma \times \sum \text { traffidoadin eachoutputport }+\eta \times \text { setupcost }+\delta \times \text { vulnerabillty }
$$

where the scaling factor is different for various managing and control strategy. The node-disjoint path finding process is summarized in [11]. The working-protection path pair forms a contour as the protection group. The successive connections will overlap their protection path by partially or fully shared with the previous protection path. Connections maybe cover many protection groups so that it will be divided as different segment automatically. Different protection scenario can be used in different group. If the connection is located in one group, the pathbased protection is adopted in principle. This default processing can be modified to the link-based or segmentbased protection due to the resource shortage. In this scenario, the connection demands will utilize all the capacity within the protection group to achieve reliable working-protection pair. If the network capacity appears insufficient, the protection path will be changed according to the result of re-computation by the nodes along the protection path, and this new protection path will form new protection group that has more capacity than previous one. If there occurs a single link or node failure, 
the protection path corresponding to the failed working path will be replaced along the alternating path in the protection group. Since the connection provisioned is short-lived, the group boundary will change dynamically. Nodes within the same group will share all the resource in a group. Sometimes the groups will be merged not because the resource insufficiency but when some connections or groups intersect with the others. In these cases the shared protection capacity will be re-computed to satisfy all the working paths in the merged group.

\section{Simulation results}

We evaluate the effectiveness of the proposed mechanisms by performing extensive simulations. The characteristics of simulated networks considered here are listed in Table 1. A duplex link is comprised of two simplex links in the opposite directions, and each simplex link is assumed to have 16 wavelengths. The connection requests arrive at a node as a Poisson process with exponentially distributed holding time with unit mean. We use 4 metrics: connection blocking probability, resource utilization, restoration time and recovery ratio to measure the performance of the proposed scenarios. We compare the performance of our method with that of an algorithm that does not use any spare lightpath. The blocking probability is evaluated by the success ratio that the spare path could be designated during working path establishment phase. The attribute of the traffic will be verified first to classify its priority, spare path assignment procedure will continue if it is a premium-class reliabilityrequired connection request. If the process failed to assign a proper backup route then block this request. But this block will trigger the resource aggregating process of the protection group to collect and update the existing resource allocation to prepare for successive request. The resource utilization is examined limited within one protection group and expect that the fully exploitation can be achieved. The restoration time is evaluated by summing several factors including failed point to end node notification time, path switching time and spare node setup time. We assign some test values to different factors in cost function to incorporate practical values in the actual network.

The blocking probability versus loading factor is plotted in Figure 3. From this figure we note that this value can be adjusted dynamically according to the selection strategy of protection group, and it is higher when protection group size reduces because of the heavy traffic contention in the same group area.
The wavelength utilization ratio versus loading factor is also plotted in Figure 3. There divided the ratio into 2 types: local and global. We only present local utilization in the figure. From there We can see that the wavelength resource is exhausted soon in each protection group, which makes the expansion process is activated. This ratio

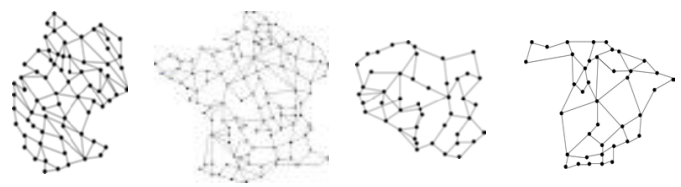

Table 1. Sample network topology information and statistical simulation results.

\begin{tabular}{|c|c|c|c|c|}
\hline $\begin{array}{c}\text { Target } \\
\text { network }\end{array}$ & $\begin{array}{c}\text { Node } \\
\text { number }\end{array}$ & $\begin{array}{c}\text { Edge } \\
\text { number }\end{array}$ & $\begin{array}{c}\text { Average } \\
\text { node } \\
\text { degree }\end{array}$ & $\begin{array}{c}\text { Average } \\
\text { group } \\
\text { size }\end{array}$ \\
\hline (a) Germany & 73 & 130 & 3.56 & 9 \\
\hline (b) French & 122 & 214 & 3.51 & 12 \\
\hline (c) Poland & 47 & 70 & 2.98 & 8 \\
\hline (d) Spain & 40 & 60 & 3.0 & 7 \\
\hline
\end{tabular}

is not only relative to the distribution of the traffic demand but also to the node connectivity. From the result of restoration ime versus loading factor, we note that the setup time of the reliable connection is longer in average due to the node-disjoint paths finding process. As expected that when load is increasing, the exploitation of network resource tends to global accessing which then makes the result approaching to path-based protection strategy.

We also list the average group size (in hop length) in Table 1. The local resource exhaustion appears in dense topology more frequently, and since we generate the connection requests in any length, so it is beneficial to dense network for more spare capacity can be exploited in the group. For evaluating the recovery ratio we examine the restorability metric by inserting 20 failures distributed in random manner and then inspect the failure recovery capability for all affected traffics. The dense networks have significant superior since the larger successful rerouting possibility in the original or merged group.

\section{Conclusion}

This paper studied the group protection based restoration heuristics in optical WDM networks. We discuss both the static and dynamic grouping scenarios. By these group models we can handle single/multiple link/node failures occurred in the networks. Results 
demonstrate that using a combination of the adaptive group-based strategy with appropriate chosen cost function based on consideration of factors about network capacity will result in better performance for resource utilization on failure restoration.

\section{Acknowledgement}

This research was supported in part by the Development of Communication Software Core Technology project of Institute for Information Industry and sponsored by MOEA, R.O.C.

\section{References}

[1] H. Zang, J. P. Jue, and B. Mukherjee, "A review of routing and wavelength assignment approaches for wavelengthrouted optical WDM networks," Optical Networks Magazine, vol. 1, no. 1, January 2000, pp. 47-60.

[2] B. T. Doshi, S. Dravida, P. Harshavardhana, O. Hauser, and Y. Wang, "Optical network design and restoration," Bell Labs. Technical Journal, Jan.-Mar. 1999, pp. 58-84.

[3] S. Ramamurthy and B. Mukherjee, "Survivable WDM mesh networks, Part II--Restoration,” Proc. ICC, Vol. 3, 1999, pp. 2023-2030.

[4] S. Ramamurthy and B. Mukherjee, "Survivable WDM mesh networks, Part I-protection," Proc. IEEE INFOCOM, 1999, pp. 744-751.

[5] G. Mohan and Arun K. Somani, "Routing Dependable Connections with Specified Failure Restoration Guarantees in WDM Networks," in Proc. IEEE INFOCOM, vol. 3, 2000, pp. $1761-1770$

[6] V. Anand, et al., "Sub-path protection: a new framework for optical layer survivability and its quantitative evaluation," UB CSE Technical Report, Jan. 2002.

[7] C. Qu, et al., "Sub-path protection for scalability and fast recovery in WDM mesh networks," Proc. OFC' 02', Anaheim, CA, Mar. 2002, pp. 495-497.

[8] P. H. Ho and T. M. Hussein, "A framework for serviceguaranteed shared protection in WDM mesh networks," IEEE Commun. Mag., Feb. 2002, 40(2), pp. 97-103.

[9] M. Medard, S. G. Finn and R. A. Barry, "WDM loop back recovery in mesh networks," IEEE INFOCOM, 1999, PP. 744-751.

[10] W. D. Grover and D. Stamatelakis, "Cycle-oriented distributed preconfiguration: ring-like speed with mesh-like capacity for self-planning network restoration,” IEEE ICC, 1998, pp. 537-543.

[11] C. S. Ho, Ing-Yi Chen and S. Y. Kuo, "A novel restoration scheme using protection domain under dynamic traffic demands in WDM networks," ICOIN'03, Jeju, Korea, Feb. 2003, pp. 108-117.
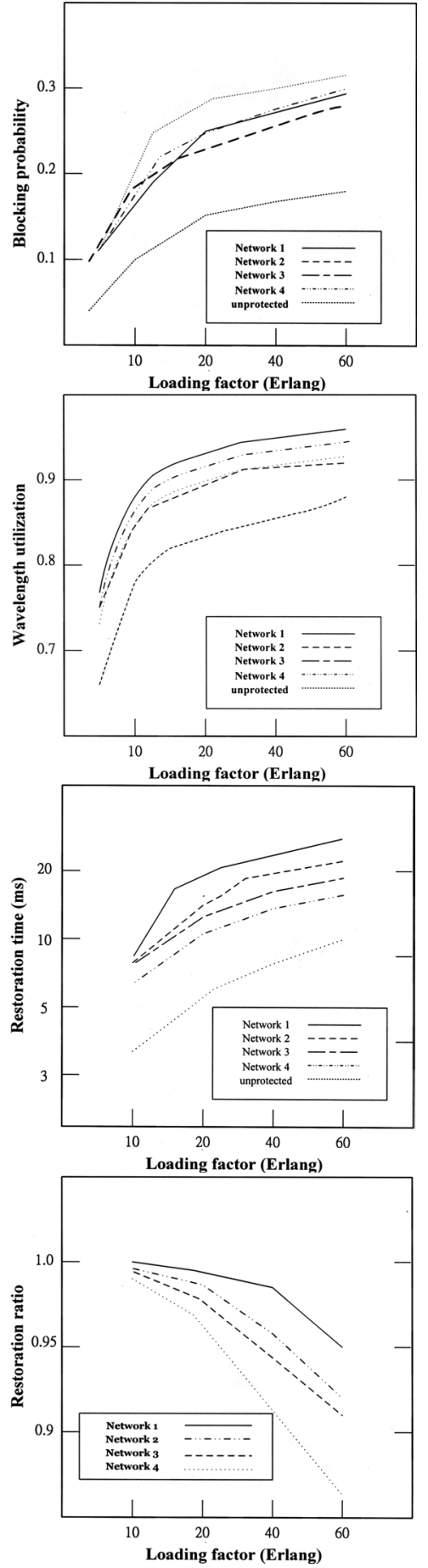

Figure 3. Various simulation results for different parameters . 